\title{
Manual de normas y procedimientos administrativos y contables para la certificación de fiel cumplimiento
}

Manual of administrative and accounting standards and procedures for the certification of faithful compliance with cooperatives

\begin{tabular}{|rrr|}
\hline Dayana Chen & Yareliz Díaz & Yenny Griman \\
\hline $\begin{array}{r}\text { dayana_chen@hotmail.com } \\
\text { ORCID: 0000-0003-1277-2764 }\end{array}$ & ORCID: 0000-0003-2548-207X & ORCID: 0000-0001-9388-7832 \\
& & Universidad de Carabobo, Venezuela
\end{tabular}

Artículo recibido en enero 2019 / Arbitrado en febrero 2019 / Publicado en mayo 2019

\section{RESUMEN}

Palabras clave:

ABSTRACT

Keywords:
Las empresas buscan mejorar sus procesos administrativos y contables, con la finalidad de cumplir con lo estipulado por los organismos supervisores; esta investigación tiene por objeto presentar el diseño de un manual de normas y procedimientos contables y administrativos para optimizar los procesos de las Cooperativas y obtener el certificado de fiel cumplimiento. Para mejores resultados en las operaciones se realiza el estudio de forma sistemática, mediante un proyecto factible, sustentado en una investigación de campo descriptiva y documental, utilizando como técnicas de recolección de datos, la encuesta y observación directa, preguntas y lista de cotejo, con alternativas de respuestas cerradas, la población estuvo conformada por los empleados. Se evidenció que la cooperativa no cuenta con inducciones escritas, documentados y normas que establezcan las operaciones de la misma, no poseen la estructura organizacional. Por consiguiente se propone el manual de normas y procedimientos, que permita optimizar su funcionamiento y la obtención de los certificados pertinentes.

Cooperativa, contabilidad, Manual de normas y procedimientos, SUNACOOP

Companies seek to improve their administrative and accounting processes, in order to comply with what is stipulated by supervisory agencies; The purpose of this research is to present the design of a manual of accounting and administrative rules and procedures to optimize the processes of the Cooperatives and obtain the certificate of faithful compliance. For better results in operations, the study is carried out systematically, through a feasible project, based on a descriptive and documentary field investigation, using as data collection techniques, the survey and direct observation, questions and checklist, with alternatives of closed answers, the population was made up of employees. It was evident that the cooperative does not have written, documented inductions and norms that establish its operations, they do not have the organizational structure. Therefore, the manual of rules and procedures is proposed, which allows optimizing its operation and obtaining the relevant certificates. 


\section{INTRODUCCIÓN}

En Latinoamérica es cada día más evidente la decadencia de los sistemas productivos, el fracaso tanto de las pequeñas como medianas empresas, el empobrecimiento de algunos sectores, la disminución de los salarios de los trabajadores y la extensión de la pobreza, en consecuencia se hace inaplazable la implementación de una alternativa de desarrollo que incorpore a la población a los procesos productivos, de manera que puedan actuar como sujetos de su propio desarrollo humano y por esa vía avanzar y contribuir en la solución de los problemas sociales y económicos de la región, entre esas alternativas se encuentran las Asociaciones Cooperativas, que no son más que organizaciones voluntarias y abiertas que crean y desarrollan sus propias culturas empresariales.

Vale la pena señalar, que en Venezuela las cooperativas presentaban un beneficio particular al encontrarse exoneradas del pago de impuestos nacionales directos, tasas, contribuciones especiales y derechos registrables así como lo establecía la ley de ISLR en gaceta Oficial No 38.628 del 16 de febrero de 2007, articulo 14; el gobierno Nacional les da prioridad ante licitaciones con el Estado, debido a que son asociaciones creadas para el beneficio de personas de medianos recursos con el interés de invertir y surgir ante la sociedad, en las cooperativas cada miembro tiene un solo voto independientemente de la cantidad de cuotas sociales que posea, el capital es indeterminado, las cuotas de participación son intransferibles y solo deben ser negociadas entre cooperadores, por lo que era atractivo para cualquier inversionista la constitución de una cooperativa antes que la de una compañía anónima.

Así pues, luego del anuncio presidencial realizado en el marco de la Ley Habilitante para el año 2013-2014, publicada en la Gaceta Oficial de la República Bolivariana de Venezuela $N^{\circ} 6.152$ de fecha 18 de noviembre de 2014, se establece que las Asociaciones Cooperativas además de declarar deben pagar los tributos correspondientes, quedando derogado el Decreto de la Ley de Impuesto Sobre la Renta publicada en la Gaceta Oficial $N^{\circ}$ 38.628 de fecha 16 de febrero de 2007, quitándole a estas asociaciones el beneficio particular que las hacía más atractiva y disminuyendo la constitución de estas asociaciones.

Cabe destacar, que la COOPERATIVA LOS CONSTRUCTORES 704,RL ubicada en Valencia Estado Carabobo se encuentra insolvente y sujeta a ser multada por los distintos organismos gubernamentales debido a que desde la fecha de constitución nunca han llevado control de los balances de comprobación, no realizan el vaciado de información financiera y administrativa en los libros obligatorios, no cuentan con la propuesta de proyectos sociales exigido en el reglamento de la (SUNACOOP), no realizan conciliaciones bancarias, mucho menos cumplen con los libros de compras y 
ventas que soportan las declaraciones de I.V.A realizadas ante el SENIAT, violando así lo establecido en el artículo 53 de la Ley de Asociación Cooperativa, e impidiendo realizar la solicitud del certificado de fiel cumplimiento.

Aunado a esto, es importante dar a conocer que La Superintendencia Nacional de Cooperativas (SUNACOOP). Es un brazo de acción del Ministerio del Poder Popular para las Comunas y los Movimientos Sociales, que tiene como objetivo establecer las normas generales para la organización y funcionamiento de las cooperativas mediante el control y la fiscalización para dar cumplimiento con lo establecido en la Ley Especial de Asociaciones Cooperativas, es el ente encargado de emitir un certificado de fiel cumplimiento.

Debe señalarse, que el Certificado de Fiel Cumplimiento antes mencionado es necesario para la inscripción ante el Instituto Nacional de Capacitación y Educación Socialista (INCES), generando a su vez la solvencia laboral ante el Ministerio del Poder Popular para el Trabajo y Seguridad Social (MINPPTRAS), necesaria para la participar en licitaciones con el gobierno mediante el Registro Nacional de Contratista (RNC), realizar cualquier operación ante el Registro Mercantil, entre otras.

Así mismo, desde el punto de vista administrativo y contable no se explica cómo esta cooperativa hasta la fecha tiene un nivel de contratación alto ante el gobierno (Información verificada y suministrada por el R.N.C), si hasta la actualidad no ha cumplido con lo exigido, carece de proyectos sociales, no presenta la memoria y cuenta de cada una de sus instancias: Administrativas, educativas, de control y evaluación; es posible pensar que la (SUNACOOP) no ejerce su función como se debe.

En vista de la problemática expuesta, esta investigación tiene como propósito diseñar un manual de normas $y$ procedimientos administrativo contable que describa de manera detallada, específica y estructurada, las actividades y funciones que se deben realizar diariamente ante la recepción de documentos administrativos, mejorando las normas de control interno utilizadas actualmente por la asociación y promoviendo así el buen uso de los implementos y el cumplimiento de las normativas establecidas en la ley.

Es importante resaltar, que los manuales constituyen una fuente formal y permanente de información y orientación, presentan una visión integral de cómo opera una unidad responsable de la ejecución de un procedimiento determinado, logran darle continuidad a la organización independientemente de los cambios que puedan surgir en la rotación de personal, permiten dar inducción y orientación al nuevo ingreso a fin de facilitarles su integración en la organización, facilitan las labores de auditoría, control interno y permiten detectar la duplicidad de funciones.

Cabe considerar, que el manual de normas y procedimientos no solo mejorará el funcionamiento interno de la cooperativa, sino que facilitará la obtención semestral del 
certificado de fiel cumplimiento emitido por la (SUNACOOP), el cual es necesario para formalizar la inscripción de la cooperativa ante el Instituto Nacional de Capacitación y Educación Socialista (INCES), Solicitar la Solvencia laboral ante el Ministerio del Poder Popular para el Trabajo y Seguridad Social (MINPPTRAS), dando cumplimiento a las contribuciones parafiscales establecidas en la Ley, la participación en Contrataciones y licitaciones ante el Estado aumentarían, originando mayores ingresos a la asociación.

Además, ante una posible supervisión de entes gubernamentales se evitarían sanciones, ya que sus registros y soportes financieros estarán en óptimas condiciones para cualquier evaluación; los asociados podrán establecer toma de decisiones claras y oportunas ante información económica y financiera lo más confiable y segura posible.

Así mismo, el alcance de esta investigación no se limita únicamente a la cooperativa en estudio, sino que además sirve de ejemplo a otras asociaciones cooperativas que presentan inconvenientes similares, beneficiará a los clientes ya que contarán con un servicio eficiente y a los proveedores a quienes se les presentará información estable de la asociación con miras a la obtención de créditos.

\section{MATERIALES Y METODO}

La presente investigación busca dar una propuesta para la elaboración de un manual de normas y procedimientos administrativos y contables que permitan la obtención de un certificado de fiel cumplimiento para la cooperativa "Los Constructores 704, RL", la presente investigación se enmarca dentro de la modalidad de proyecto factible, el cual según la Universidad Pedagógica Experimental Libertador (UPEL, 2010), establece que "la investigación, elaboración Viable para solucionar, requerimientos o necesidades de organizaciones o grupos sociales..." (p.16); Donde considerando los objetivos planteados la presente investigación es de nivel descriptivo, por ende, su propósito es precisar en forma detallada la naturaleza del problema en el momento actual, lo cual conlleva a realizar un análisis cuantitativo de los resultados para interpretar el significado. Es una investigación de campo no experimental, ya que los datos se recolectaron directamente de la realidad y se aplicará el instrumento.

Para el estudio en cuestión la población y la muestra que se utilizará está conformada por 8 personas del personal administrativo contable de la Cooperativa Los Constructores 704, R.L. Toda investigación requiere del manejo $\mathrm{y}$ conocimiento de información previa que permitirá poner en marcha el estudio, para eso es necesario emplear una serie de técnicas e instrumentos, en este caso la técnica que se utilizó para recolectar la información fue la encuesta y la observación directa, la misma permitirá diagnosticar la situación actual y su ventaja fue en que son los mismos actores sociales quienes aportaron los datos relativos a sus conductas, opiniones, deseos actitudes. Esto permite tener una visión general del 
problema existente, así como recabar la información necesaria para elaborar las estrategias.

Aplicando como instrumento un cuestionario a los departamentos de administración y contabilidad, compuesto por ítems que se derivarán de los objetivos de la Investigación; su estructura y forma fueron representadas a través de la redacción de preguntas, con alternativas de respuesta cerradas tipo dicotómicas, en las cuales solo se puede marcar una opción por ítems. Este cuestionario está conformado por dieciséis (13) ítems.

\section{RESULTADOS Y DISCUSIÓN}

En un principio se procedió a obtener la información a través de las siguientes técnicas: la observación directa no participante y la revisión documental, los cuales se llevaron a cabo mediante visitas realizadas a la Cooperativa. Para cumplir con lo establecido en la técnica de observación no participante se usó una guía constituida por una lista de cotejo. Se evidenció que la cooperativa no cuenta con inducciones escritas, documentados y normas que establezcan las operaciones de la misma, no poseen la estructura organizacional con la descripción de cada uno de los cargos, delegando las funciones y responsabilidad de cada cargo.
En tal sentido, se puede evidenciar que no se cuenta con manual de normas y procedimientos documentados que establezcan los parámetros y actividades en los procesos de la organización contable y administrativa que logren cumplir con los requisitos exigidos para la solicitud $y$ obtención del fiel cumplimiento ante la Superintendencia Nacional de Cooperativas (SUNACOOP).

A pesar de lo descrito anteriormente, los empleados cumplen con algunas medidas para realizar las actividades como se deben en el proceso administrativo y contable, pero se realiza de manera informal y no por escrito efectuado bajo el criterio del personal y no bajo el criterio que debe ser establecido en el control interno de la cooperativa.

De igual manera, se pudo observar debilidades en el control interno en cuanto los soportes y formatos necesarios para llevar el control de las partidas como cuentas por cobrar y cuentas por pagar por nombrar algunas, las cuales son importantes como todas las demás partidas para tener conocimiento objetivo y veraz y estar al tanto de la parte operativa y del funcionamiento de la cooperativa, clave fundamental que puede afectar la toma de decisiones. A continuación se pueden observar los resultados obtenidos una vez aplicado el instrumento. 
Tabla 1. Ítem ¿Desde el inicio de las operaciones de la Cooperativa los constructores 704, R.L, se realizan las declaraciones exigidas por el SENIAT?

\begin{tabular}{lcc}
\hline \multicolumn{1}{c}{ Alternativas } & Frecuencia & Porcentajes \\
\hline $\mathrm{Si}$ & 2 & $25 \%$ \\
No & 2 & $25 \%$ \\
Algunas veces & 4 & $50 \%$ \\
Total & $\mathbf{8}$ & $\mathbf{1 0 0 \%}$ \\
\hline
\end{tabular}

Fuente: Chen. Díaz. Griman (2017)

Interpretación: En la tabla N. ${ }^{\circ} 1$ se realizó una muestra del $100 \%$ de los encuestados, obteniendo como resultado que el $50 \%$ de la población coincide que la Cooperativa los constructores 704, R.L no realiza periódicamente las declaraciones exigidas por el SENIAT, trayendo como consecuencia, la ausencia del soporte necesario exigido por la SUNACOOP para la obtención del certificado de fiel cumplimiento, además la Cooperativa puede ser multada por el SENIAT, por no realizar las declaraciones exigidas en los periodos establecidos por el ente rector, trayendo como consecuencia desembolso por parte de la asociación cooperativa para el pago de dicha multa, no poder participar en licitaciones por parte de las instituciones públicas, bajando así su nivel de contratación y afectando los ingresos de la misma.

Tabla 2. Ítem ¿Considera usted que posee los conocimientos de las normas y procedimientos para registrar las operaciones diarias que efectúan en su departamento?

\begin{tabular}{lcc}
\hline \multicolumn{1}{c}{ Alternativas } & Frecuencia & Porcentajes \\
\hline $\mathrm{Si}$ & 3 & $37 \%$ \\
No & 0 & $0 \%$ \\
Algunas veces & 5 & $63 \%$ \\
Total & $\mathbf{8}$ & $\mathbf{1 0 0 \%}$ \\
\hline
\end{tabular}

Fuente: Chen. Díaz. Griman (2017)

Interpretación: Con base en los resultados obtenidos, se puede demostrar que el $63 \%$ de los encuestados coinciden que veces poseen algunos los conocimientos en cuanto a normas y procedimientos necesarios para el registro eficaz de sus labores diarias, este resultado deja evidencia de que los empleados de los departamentos administrativos y contables, no siempre están seguros de como registrar sus operaciones diarias, trayendo como consecuencia error en los registros contables, que a su vez afectan la veracidad de los estados financieros de la Cooperativa los constructores 704 R.L. 
Tabla 3. Ítem ¿Le exigen en la Cooperativa los constructores 704 R.L llevar un control de los soportes respectivos para el registro de las operaciones?

\begin{tabular}{lcc}
\hline \multicolumn{1}{c}{ Alternativas } & Frecuencia & Porcentajes \\
\hline $\mathrm{Si}$ & 0 & $0 \%$ \\
No & 6 & $25 \%$ \\
Algunas veces & 2 & $\mathbf{7 5 \%}$ \\
Total & $\mathbf{4}$ & $\mathbf{1 0 0 \%}$ \\
\hline
\end{tabular}

Fuente: Chen. Díaz. Griman (2017)

Interpretación: las personas fraude, ya que por ejemplo si se desea emitir encuestadas coinciden en un $75 \%$ que la el pago a un proveedor y no se exige que la Cooperativa los Constructores 704 R.L no orden de pago este firmada por algún socio exige llevar un soporte respectivo de las o persona autorizada y además dejar operaciones, esto trae como consecuencia evidencia de la misma para futuras que al necesitar verificar un asiento contable auditorias, el personal encargado puede o el registro de alguna operación no se emitir pagos sin autorización o decir que esa cuente con los avales respectivos, además si emisión fue permitida verbalmente, sin dejar no se llevan los controles adecuadamente evidencia de la veracidad de la información. puede la cooperativa tener un riesgo de

Tabla 4. Ítem ¿Cuentan con normas internas en la cooperativa los constructores $704 \mathrm{RL}$ para el registro de las operaciones diarias?

\begin{tabular}{lcc}
\hline \multicolumn{1}{c}{ Alternativas } & Frecuencia & Porcentajes \\
\hline $\mathrm{Si}$ & 0 & $0 \%$ \\
No & 8 & $100 \%$ \\
Algunas veces & 0 & $0 \%$ \\
Total & $\mathbf{8}$ & $\mathbf{1 0 0 \%}$ \\
\hline
\end{tabular}

Fuente: Chen. Díaz. Griman (2017)

Interpretación: de acuerdo a los resultados obtenidos se puede observar que el $100 \%$ de los encuestados indican que en la Cooperativa los Constructores 704 R.L no cuentan con normas internas para el registro de las operaciones diarias, pudiendo ocasionar duplicidad u omisión en el registro de información administrativa y contable. 
Tabla 5. Ítem ¿Realizan periódicamente seguimiento de las operaciones diarias, con la finalidad de enmendar errores?

\begin{tabular}{lcc}
\hline \multicolumn{1}{c}{ Alternativas } & Frecuencia & Porcentajes \\
\hline $\mathrm{Si}$ & 0 & $0 \%$ \\
No & 6 & $5 \%$ \\
Algunas veces & 2 & $\mathbf{2 5 \%}$ \\
Total & $\mathbf{8}$ & $\mathbf{1 0 0 \%}$ \\
\hline
\end{tabular}

Fuente: Chen. Díaz. Griman (2017)

Interpretación: Con respecto al ítem $N^{\circ} 5$ se puede afirmar que un $75 \%$ de la población concuerda que solo algunas veces se realizan evaluaciones de las operaciones diarias registradas, con la finalidad de enmendar errores en el registro de las mismas; lo que quiere decir, que al no realizar la revisión periódica de las transacciones, la cooperativa confía en la evaluación y registro de ciertas operaciones importantes la empresa, por ende solo se dedica a la supervisión de otras operaciones.

Tabla 6. Ítem ¿Realizan cursos o actualizaciones periódicas, que los ayuden a estar al día con las exigencias emitidas por los entes regulatorios?

\begin{tabular}{lcc}
\hline Alternativas & Frecuencia & Porcentajes \\
\hline $\mathrm{Si}$ & 0 & $0 \%$ \\
No & 8 & $100 \%$ \\
Algunas veces & 0 & $0 \%$ \\
Total & $\mathbf{8}$ & $\mathbf{1 0 0 \%}$ \\
\hline
\end{tabular}

Fuente: Chen. Díaz. Griman (2017)

Interpretación: En relación a la tabla No 6 los resultados arrojaron que el 100\% de los encuestados concuerdan que la Cooperativa los Constructores 704 R.L no realiza cursos periódicos que ayuden a sus empleados a mantenerse al día con las actualizaciones exigidas por los entes gubernamentales; ocasionando que los registros de sus operaciones se estén realizando sin el debido conocimiento $y$ quizás soportadas en leyes ya derogadas y no como lo exige el ente gubernamental. 
Tabla 7. Ítem ¿Cuentan con formatos y/o documentos administrativos que ayuden en el registro de las operaciones diarias?

\begin{tabular}{lcc}
\hline \multicolumn{1}{c}{ Alternativas } & Frecuencia & Porcentajes \\
\hline $\mathrm{Si}$ & 3 & $37 \%$ \\
No & 5 & $\mathbf{6 3 \%}$ \\
Algunas veces & 0 & $\mathbf{0} \%$ \\
Total & $\mathbf{8}$ & $\mathbf{1 0 0 \%}$ \\
\hline
\end{tabular}

Fuente: Chen. Díaz. Griman (2017)

Interpretación: En cuanto a los administrativos que los ayuden en el resultados obtenidos se evidencia que el registro de las operaciones diarias; por lo $63 \%$ de la población encuestada concuerda que conlleva que a su vez no se les exija que la Cooperativa los constructores 704 soporte de las transacciones que avalen los $\mathrm{RL}$ carece de formatos $\mathrm{y} / \mathrm{o}$ documentos registros de sus operaciones diarias.

Tabla 8. Ítem ¿Cuenta la Cooperativa los Constructores 704 R.L con los soportes necesarios para respaldar la información que presenta actualmente los estados financieros?

\begin{tabular}{lcc}
\hline \multicolumn{1}{c}{ Alternativas } & Frecuencia & Porcentajes \\
\hline Si & 3 & $37 \%$ \\
No & 0 & $0 \%$ \\
Algunas veces & 5 & $63 \%$ \\
Total & $\mathbf{8}$ & $\mathbf{1 0 0 \%}$ \\
\hline
\end{tabular}

Fuente: Chen. Díaz. Griman (2017)

Interpretación: En cuanto a los resultados obtenidos, se prueba que los empleados del área administrativa contable concuerdan en un $63 \%$ que los registros presentados en los estados financieros de la Cooperativa los constructores 704, R.L carecen de soportes que los avalen, perdiendo así objetividad la información recopilada y a su vez corriendo el riesgo la cooperativa de ser multados y cerrados, ante una supervisión por entes gubernamentales. 
Tabla 9. Ítem ¿Considera usted que con un manual de normas y procedimientos en la Cooperativa los constructores 704 R.L, se optimizaran los procesos del departamento administrativo y contable?

\begin{tabular}{lcc}
\hline \multicolumn{1}{c}{ Alternativas } & Frecuencia & Porcentajes \\
\hline $\mathrm{Si}$ & 8 & $100 \%$ \\
No & 0 & $0 \%$ \\
Algunas veces & 0 & $0 \%$ \\
Total & $\mathbf{8}$ & $\mathbf{1 0 0 \%}$ \\
\hline
\end{tabular}

Fuente: Chen. Díaz. Griman (2017)

Interpretación: Como se evidencia en el cuadro anterior el $100 \%$ de los encuestados coinciden que con un manual de normas y procedimientos administrativo contable se optimizaran las operaciones que se realizan diariamente en la Cooperativa los Constructores 704 R.L

Tabla 10. Ítem ¿Existe información escrita sobre los procedimientos administrativos y contables que deben llevarse en la Cooperativa los constructores 704 R.L?

\begin{tabular}{lcc}
\hline \multicolumn{1}{r}{ Alternativas } & Frecuencia & Porcentajes \\
\hline $\mathrm{Si}$ & 0 & $0 \%$ \\
No & 8 & $100 \%$ \\
Algunas veces & 0 & $0 \%$ \\
Total & $\mathbf{8}$ & $\mathbf{1 0 0 \%}$ \\
\hline
\end{tabular}

Fuente: Chen. Díaz. Griman (2017)

Interpretación: Según la opinión cuenta procedimientos administrativos y emitida por la muestra de estudio, el $100 \%$ contables, escritos que indiquen a sus de los encuestados coinciden en que la analistas como registrar las operaciones y Cooperativa los Constructores 704 R.L no llevar a cabo sus labores de manera eficaz. 
Tabla 11. Ítem ¿Existe segregación de funciones entre los departamentos de la Cooperativa los constructores 704

\begin{tabular}{lcc}
\hline \multicolumn{1}{c}{ Alternativas } & Frecuencia & Porcentajes \\
\hline $\mathrm{Si}$ & 2 & $25 \%$ \\
$\mathrm{No}$ & 4 & $50 \%$ \\
Algunas veces & 2 & $25 \%$ \\
Total & $\mathbf{4}$ & $\mathbf{1 0 0 \%}$ \\
\hline
\end{tabular}

Fuente: Chen. Díaz. Griman (2017)

Interpretación: Para este ítem, se puede observar que el $50 \%$ los encuestados concuerdan, que la Cooperativa los Constructores 704 R.L no cuenta con segregación de funciones en su departamento administrativo y contable, por lo cual se puede deducir que existen posibilidades de riesgo ya que una misma persona realiza labores de aprobación, ejecución entre otras actividades, por lo que no se estaría evaluando si los registros corresponden, si la emisión de pagos corresponden al beneficiario, si el monto a cancelar es el correcto y además si la cuenta contable a para el registro de la operación es la correcta.

Tabla 12. Ítem ¿Están sus deberes y responsabilidades laborales establecidas de manera formal?

\begin{tabular}{lcc}
\hline \multicolumn{1}{c}{ Alternativas } & Frecuencia & Porcentajes \\
\hline $\mathrm{Si}$ & 2 & $25 \%$ \\
No & 3 & $37 \%$ \\
Algunas veces & 3 & $38 \%$ \\
Total & $\mathbf{8}$ & $\mathbf{1 0 0 \%}$ \\
\hline
\end{tabular}

Fuente: Chen. Díaz. Griman (2017)

Interpretación: Luego de aplicado el cuestionario a la población de estudio, se observa que el $38 \%$ de los encuestados, concuerdan que en la Cooperativa los Constructores 704 R.L tienen algunas actividades establecida de manera formal, mientras que el resto de las actividades que se les asignan, pero no existe evidencia formal que sean de su competencia llevarlas a cabo. 
Tabla 13. Ítem ¿Conoce usted la finalidad de las labores que realiza para las operaciones administrativa y contable de la empresa?

\begin{tabular}{lcc}
\hline \multicolumn{1}{c}{ Alternativas } & Frecuencia & Porcentajes \\
\hline $\mathrm{Si}$ & 4 & $50 \%$ \\
$\mathrm{No}$ & 0 & $0 \%$ \\
Algunas veces & 4 & $50 \%$ \\
Total & $\mathbf{8}$ & $\mathbf{1 0 0 \%}$ \\
\hline
\end{tabular}

Fuente: Chen. Díaz. Griman (2017)

Interpretación: $A$ través de la tabla anterior se demuestra que el 50 por ciento de los encuestados afirman que conoce la finalidad de las operaciones que realizan diariamente para la en la Cooperativa los constructores 704 R.Li pero también tenemos como resultado otro 50 por ciento no tiene el conocimiento pleno, por lo que nos permite afirmar que estos empleados pueden presentar fallas en los registros, ya que no saben el fin de los mismos en la empresa, ocasionando también que a su vez los estados financieros no estén del todo correctos.

\section{CONCLUSIÓN}

Después de realizar un estudio preliminar al departamento de administración y contabilidad de la Cooperativa Los Constructores 704, R.L., se llega a la conclusión que la cooperativa no cuenta con procedimientos, normas y políticas administrativas ni contables que estén plasmadas por escrito en las políticas de la Cooperativa, sin embargo se evidencia que los empleados establecen sus procedimientos en ambos departamentos, lo que lleva a indagar que los mismos fueron establecidos en forma verbal e informal; los cuales tienen debilidades por la manera de cómo se efectúan tales procedimientos, la información obtenida da el indicio que la cooperativa no efectúa correctamente los procesos necesarios para cumplir con los requisitos exigidos por la SUNACOOP para la obtención del certificado de fiel cumplimiento.

Por otro lado, la falta de formatos para la supervisión de los procesos administrativos y contables que son necesarios para llevar mensualmente un registro de las conciliaciones bancarias, balances de comprobación, nominas, facturación, cuentas por cobrar, cuentas y efectos por pagar, gastos generales, etc, y la segregación de funciones contribuye aún más a la debilidad detectada. Es importante resaltar que la cooperativa cuenta con recursos humanos que están dispuestos aumentar y mejorar su capacidad laboral, la gerencia está abierta aceptar sugerencias para el mejoramiento del proceso interno de los departamentos administrativo y contable así 
como la aceptación de la implementación del manual debidamente documentado con todo lo relacionado a los departamentos afectados, sus funciones y la delegación de responsabilidades en cada uno los empleados de los respectivos departamentos.

Partiendo de todo lo señalado anteriormente, se demuestra que la necesidad de la elaboración de un manual de normas y procedimientos contables y administrativos describiendo los pasos a seguir para la solicitud del certificado de fiel cumplimiento, el cual es importante para mantener a flote las operaciones productivas de las cooperativas; además de cumplir con las políticas y normas financieras, administrativas y contables demostrado en los estados financieros y las conciliaciones bancarias que es uno de los principales requisitos para la solicitud. $Y$ a su vez:

- Optimizar la formación del personal que laboran en los departamentos de contabilidad y administración en cuanto a los procesos y las actividades que se deben ejecutar correctamente en cada uno de los departamentos.

- Delegar funciones en los cargos que ocupan cada uno de los empleados de los diferentes departamentos con la finalidad de segregar actividades.

- Incorporar en el presupuesto una reserva destinada al adiestramiento del personal del área administrativa y contable.
- Determinar los parámetros de supervisión y control para que desde el momento en que se implemente el manual todos los empleados cumplan con lo que establece el mismo.

- Realizar supervisiones periódicas para lograr la eliminación de la debilidad detectada y cumplir con las correcciones pertinentes y necesarias para lograr los cambios esperados contemplados en el manual.

- Realizar una revisión de la información que presentan actualmente los estados financieros, para verificar la veracidad de la misma y realizar los ajustes pertinentes de conseguir alguna información mal registrada.

\section{REFERENCIAS}

Hernández S, Fernández C, Baptista P (2014), Metodología De La Investigación. 6ta Edición. Interamericana de Editores, S.A. México Lugo J, (2013) Propuesta de un manual de normas y procedimientos administrativos y contables aplicados a la empresa Riviera del Guarapiche del Municipio Maturin del Estado Monagas. Trabajo de Grado. Universidad de Oriente

Cuerva Y., (2013), Manual de normas y procedimientos contables en el proceso de compras utilizando el sistema Profit en la empresa Ipeca Construcciones, C.A. Trabajo de Grado. Universidad de Carabobo 
Colmenares, Figueredo (2012), Diseño De Un Manual de Normas y Procedimientos Basado En La Filosofía De Las 5 s's Como Herramienta De Mejoramiento Continuo $Y$ Fortalecimiento Económico Para La Empresa Portugalia Import, C.A. Trabajo de Grado. Universidad de Carabobo

Nunes L, Quintero J y Quintero S, (2014), Manual De Normas Y Procedimientos para el área de nóminas en la almacenadora San Diego, C.A. Trabajo de Grado. Universidad de Carabobo

Universidad Pedagógica Experimental Libertador UPEL (2010). Normas Para La Elaboración, Presentación Y Evaluación De Los Trabajos Especiales De Grado. Ediciones UNA

Balestrini M, (2006), Como se elabora el proyecto de investigación, 7ma Edición Venezuela. BL Consultores y Asociados

Hurtado y Toro, (2001), Paradigma y métodos de investigación en tiempo de cambio, 5ta Edición, Episteme Consultores Asociados, C.A

Hurtado J, (2010) El proyecto de investigación Tercera edición. Editorial Sypal
Bisquerra R. (2009) Metodología de la investigación educativa. 2da edición. Editorial la Muralla.S.A

Providencia Administrativa No PA-1119-13 de SUNACOOP

Ley Especial de Asociaciones Cooperativas

Gaceta Oficial No 5.453 de la República Bolivariana de Venezuela del 24 de marzo de 2000

La ley de impuesto sobre la Renta de Gaceta Oficial No 38.628 del 16 de febrero de 2007

Gaceta Oficial de la República Bolivariana de Venezuela No. 6.152

Extraordinario, el Decreto con Rango, Valor y Fuerza de Ley de Reforma de la Ley de Impuesto Sobre la Renta

Decreto con Fuerza de Ley Especial de Asociaciones Cooperativas gaceta oficial No 37.894 de marzo 2004

SUNACOOP

(http://www.gestionparticipativa.coop/p ortal/index.php?option $=$ com_content $\&$ view $=$ article\&id $=391$ :requisitos- parasolicitar-los-certificados-de-fielcumplimiento-asunacoop\&catid $=37$ : getaways\&Itemid $=$ 364.) 